Francisco J. CARo-Lopera (Guanajuato)

José A. Díaz-García (Saltillo)

Graciela GonzÁlez-Farías (Guanajuato)

\title{
A FORMULA FOR JACK POLYNOMIALS OF THE SECOND ORDER
}

Abstract. This work solves the partial differential equation for Jack polynomials $C_{\kappa}^{\alpha}$ of the second order. When the parameter $\alpha$ of the solution takes the values $1 / 2,1$ and 2 we get explicit formulas for the quaternionic, complex and real zonal polynomials of the second order, respectively.

1. Introduction. The computation of Jack polynomials has undergone a vertiginous development in the last few years: see Goulden and Jackson (1996), Sawyer (1997), Koev (2004), Koev and Edelman (2006) and Dumitriu et al. (2005), among many others. Before Jack polynomials, the real and complex zonal polynomials have been studied extensively in the statistical literature. Important open problems concerning zonal polynomials could be handled with Jack polynomials theory, using the fact that the zonal polynomials of a symmetric matrix and the zonal polynomials of a hermitian matrix are Jack polynomials for $\alpha=2$ and $\alpha=1$, respectively; see James (1964), James (1968), Khatri (1970), Muirhead (1982), Díaz-García and Caro-Lopera (2006) and Díaz-García and Caro-Lopera (2007), among many others.

It is known that the real and complex zonal polynomials are eigenfunctions of the Laplace-Beltrami operator. The resulting partial differential equation leads to a recurrence relation for their coefficients and so the polynomials can be computed. A few explicit formulae to calculate the Jack polynomials appear in the literature; specifically, in the real case, i.e. when

2000 Mathematics Subject Classification: 05E05, 35M99, 33C05.

Key words and phrases: Jack functions, Jack polynomials, Laplace-Beltrami operator, zonal polynomials, hermitian matrix, quaternionic matrix, hypergeometric differential equation. 
$\alpha=2$, James (1964, Section 9) proposes some expressions. For the same value of $\alpha$, but only for the second order, James (1968) solves the partial differential equation for real zonal polynomials.

In general, Jack polynomials are also eigenfunctions of an operator of the Laplace-Beltrami type (see Dumitriu et al. (2005, Definition 2.10)), and as before, a general recurrence relation can be derived from a partial differential equation to compute their coefficients.

Following the idea of James (1968), this work finds an explicit formula for the Jack polynomials of the second order. This is carried out by solving the general partial differential equation with a parameter $\alpha$ when two eigenvalues are considered. Taking $\alpha=1 / 2,1$, formulae for quaternionic and complex zonal polynomials of the second order are obtained, respectively. For definitions of the quaternionic zonal polynomials see Gross and Richards (1987). Also, the results derived in James (1968) for the real zonal polynomials of the second order are recovered for $\alpha=2$.

2. A formula for Jack polynomials of the second order. Let us characterize the Jack symmetric function $J_{\kappa}^{(\alpha)}\left(y_{1}, \ldots, y_{m}\right)$ of parameter $\alpha$ (see Sawyer (1997)). A decreasing sequence of nonnegative integers $\kappa=$ $\left(k_{1}, k_{2}, \ldots\right)$ with only finitely many nonzero terms is said to be a partition of $k=\sum k_{i}$. Let $\kappa$ and $\lambda=\left(l_{1}, l_{2}, \ldots\right)$ be two partitions of $k$. We write $\lambda \leq \kappa$ if $\sum_{i=1}^{t} l_{i} \leq \sum_{i=1}^{t} k_{i}$ for each $t$. The conjugate of $\kappa$ is $\kappa^{\prime}=\left(k_{1}^{\prime}, k_{2}^{\prime}, \ldots\right)$ where $k_{i}^{\prime}=\operatorname{card}\left\{j: k_{j} \geq i\right\}$. The length of $\kappa$ is $l(k)=\max \left\{i: k_{i} \neq 0\right\}=k_{1}^{\prime}$. If $l(\kappa) \leq m$, one often writes $\kappa=\left(k_{1}, \ldots, k_{m}\right)$. The partition $(1, \ldots, 1)$ of length $m$ will be denoted by $1^{m}$.

The monomial symmetric function $M_{\kappa}(\cdot)$ indexed by a partition $\kappa$ can be regarded as a function of an arbitrary number of variables such that all but a finite number are equal to 0 : if $y_{i}=0$ for $i>m \geq l(\kappa)$ then $M_{\kappa}\left(y_{1}, \ldots, y_{m}\right)=\sum y_{1}^{\sigma_{1}} \cdots y_{m}^{\sigma_{m}}$, where the sum is over all distinct permutations $\left\{\sigma_{1}, \ldots, \sigma_{m}\right\}$ of $\left\{k_{1}, \ldots, k_{m}\right\}$, and if $l(\kappa)>m$ then $M_{\kappa}\left(y_{1}, \ldots, y_{m}\right)=0$. A symmetric function is a linear combination of monomial symmetric functions. If $f$ is a symmetric function then $f\left(y_{1}, \ldots, y_{m}, 0\right)=f\left(y_{1}, \ldots, y_{m}\right)$. For each $m \geq 1, f\left(y_{1}, \ldots, y_{m}\right)$ is a symmetric polynomial in $m$ variables.

Thus the Jack symmetric functions $J_{\kappa}^{(\alpha)}\left(y_{1}, \ldots, y_{m}\right)$ with parameter $\alpha$ satisfy the following conditions:

$$
\begin{aligned}
J_{\kappa}^{(\alpha)}\left(y_{1}, \ldots, y_{m}\right) & =\sum_{\lambda \leq \kappa} j_{\kappa, \lambda} M_{\lambda}\left(y_{1}, \ldots, y_{m}\right), \\
J_{\kappa}^{(\alpha)}(1, \ldots, 1) & =\alpha^{k} \prod_{i=1}^{m}\left(\frac{m-i+1}{\alpha}\right)_{k_{i}},
\end{aligned}
$$




$$
\begin{aligned}
\sum_{i=1}^{m} y_{i}^{2} \frac{\partial^{2} J_{\kappa}^{(\alpha)}\left(y_{1}, \ldots, y_{m}\right)}{\partial y_{i}^{2}} & +\frac{2}{\alpha} \sum_{i=1}^{m} y_{i}^{2} \sum_{j \neq i} \frac{1}{y_{i}-y_{j}} \frac{\partial J_{\kappa}^{(\alpha)}\left(y_{1}, \ldots, y_{m}\right)}{\partial y_{i}} \\
= & \sum_{i=1}^{m} k_{i}\left(k_{i}-1+\frac{2}{\alpha}(m-i)\right) J_{\kappa}^{(\alpha)}\left(y_{1}, \ldots, y_{m}\right) .
\end{aligned}
$$

Here the constants $j_{\kappa, \lambda}$ do not depend on $y_{i}$ 's but depend on $\kappa$ and $\lambda$, and $(a)_{n}=\prod_{i=1}^{n}(a+i-1)$. Note that if $m<l(\kappa)$ then $J_{\kappa}^{(\alpha)}\left(y_{1}, \ldots, y_{m}\right)=0$. The conditions include the case $\alpha=0$ and then we have

$$
J_{\kappa}^{(0)}\left(y_{1}, \ldots, y_{m}\right)=e_{\kappa^{\prime}} \prod_{i=1}^{m}(m-i+1)^{k_{i}},
$$

where $e_{\kappa}\left(y_{1}, \ldots, y_{m}\right)=\prod_{i=1}^{l(\kappa)} e_{k_{i}}\left(y_{1}, \ldots, y_{m}\right)$ are the elementary symmetric functions indexed by partitions $\kappa$; if $m \geq l(\kappa)$ then $e_{r}\left(y_{1}, \ldots, y_{m}\right)=$ $\sum_{i_{1}<\cdots<i_{r}} y_{i_{1}} \cdots y_{i_{r}}$, and if $m<l(\kappa)$ then $e_{r}\left(y_{1}, \ldots, y_{m}\right)=0$ (see Sawyer (1997)).

Now, from Koev and Edelman (2006), the Jack functions $J_{\kappa}^{(\alpha)}(Y)=$ $J_{\kappa}^{(\alpha)}\left(y_{1}, \ldots, y_{m}\right)$, with $y_{1}, \ldots, y_{m}$ being the eigenvalues of the matrix $Y$, can be normalised in such a way that

$$
\sum_{\kappa} C_{\kappa}^{\alpha}(Y)=(\operatorname{tr}(Y))^{k}
$$

where $C_{\kappa}^{\alpha}(Y)$ denotes the Jack polynomials. They are related to the Jack functions by

$$
C_{\kappa}^{\alpha}(Y)=\frac{\alpha^{k} k !}{j_{\kappa}} J_{\kappa}^{\alpha}(Y)
$$

where

$$
j_{\kappa}=\prod_{(i, j) \in \kappa} h_{*}^{\kappa}(i, j) h_{\kappa}^{*}(i, j),
$$

and $h_{*}^{\kappa}(i, j)=k_{j}-i+\alpha\left(k_{i}-j+1\right)$ and $h_{\kappa}^{*}(i, j)=k_{j}-i+1+\alpha\left(k_{i}-j\right)$ are the upper and lower hook lengths at $(i, j) \in \kappa$, respectively.

Then by applying (4), we can write (3) as

$$
\begin{aligned}
\sum_{i=1}^{m} y_{i}^{2} \frac{\partial^{2} C_{\kappa}^{(\alpha)}(Y)}{\partial y_{i}^{2}}+\frac{2}{\alpha} \sum_{i=1}^{m} y_{i}^{2} \sum_{j \neq i} \frac{1}{y_{i}-y_{j}} \frac{\partial C_{\kappa}^{(\alpha)}(Y)}{\partial y_{i}} \\
=\sum_{i=1}^{m} k_{i}\left(k_{i}-1+\frac{2}{\alpha}(m-i)\right) C_{\kappa}^{(\alpha)}(Y) .
\end{aligned}
$$


3. Jack polynomials of the second order. When $m=2$ in (5) we get the partial differential equation for $C_{\kappa}^{(\alpha)}=C_{\kappa}^{(\alpha)}(Y)$,

$$
\begin{aligned}
y_{1}^{2} \frac{\partial^{2} C_{\kappa}^{(\alpha)}}{\partial y_{1}^{2}}+y_{2}^{2} \frac{\partial^{2} C_{\kappa}^{(\alpha)}}{\partial y_{2}^{2}} & +\frac{2}{\alpha} y_{1}^{2}\left(y_{1}-y_{2}\right)^{-1} \frac{\partial C_{\kappa}^{(\alpha)}}{\partial y_{1}}-\frac{2}{\alpha} y_{2}^{2}\left(y_{1}-y_{2}\right)^{-1} \frac{\partial C_{\kappa}^{(\alpha)}}{\partial y_{2}} \\
& -\left[k_{1}\left(k_{1}-1+\frac{2}{\alpha}\right)+k_{2}\left(k_{2}-1\right)\right] C_{\kappa}^{(\alpha)}=0 .
\end{aligned}
$$

Substituting $u=y_{1}+y_{2}$ and $v=y_{1} y_{2}$ in (6), we get

$$
\begin{array}{r}
\left(u^{2}-2 v\right) \frac{\partial^{2} C_{\kappa}^{(\alpha)}}{\partial u^{2}}+2 v^{2} \frac{\partial^{2} C_{\kappa}^{(\alpha)}}{\partial v^{2}}+2 u v \frac{\partial^{2} C_{\kappa}^{(\alpha)}}{\partial u \partial v}+\frac{2 u}{\alpha} \frac{\partial C_{\kappa}^{(\alpha)}}{\partial u}+\frac{2 v}{\alpha} \frac{\partial C_{\kappa}^{(\alpha)}}{\partial v} \\
-\left[k_{1}\left(k_{1}-1+\frac{2}{\alpha}\right)+k_{2}\left(k_{2}-1\right)\right] C_{\kappa}^{(\alpha)}=0 .
\end{array}
$$

Substituting now $z=u / 2 \sqrt{v}$ and $t=\sqrt{v}$ we obtain

$$
\begin{array}{r}
\left(1-z^{2}\right) \frac{\partial^{2} C_{\kappa}^{(\alpha)}}{\partial z^{2}}-t^{2} \frac{\partial^{2} C_{\kappa}^{(\alpha)}}{\partial t^{2}}-\left(\frac{2}{\alpha}+1\right) z \frac{\partial C_{\kappa}^{(\alpha)}}{\partial z}-\left(\frac{2}{\alpha}-1\right) t \frac{\partial C_{\kappa}^{(\alpha)}}{\partial t} \\
+2\left[k_{1}\left(k_{1}-1+\frac{2}{\alpha}\right)+k_{2}\left(k_{2}-1\right)\right] C_{\kappa}^{(\alpha)}=0 .
\end{array}
$$

It is easy to see that the last equation is homogeneous in $t$. Thus, by taking

$$
C_{\kappa}^{(\alpha)}=t^{k_{1}+k_{2}} f(z),
$$

we obtain an ordinary differential equation

$$
\left(1-z^{2}\right) \frac{d^{2} f}{d z^{2}}-\left(\frac{2}{\alpha}+1\right) z \frac{d f}{d z}+\left[\left(k_{1}-k_{2}\right)\left(k_{1}-k_{2}+\frac{2}{\alpha}\right)\right] f=0 .
$$

Now, taking $w=(1-z) / 2$ as the independent variable, the differential equation becomes

$$
w(1-w) \frac{d^{2} f}{d w^{2}}+\left(\frac{1}{\alpha}+\frac{1}{2}\right)(1-2 w) \frac{d f}{d w}+\varrho\left(\varrho+\frac{2}{\alpha}\right) f=0,
$$

with $\varrho=k_{1}-k_{2}>0$.

Comparing with the general hypergeometric equation

$$
w(1-w) \frac{d^{2} f}{d w^{2}}+[c-(a+b+1) w] \frac{d f}{d w}-a b f=0,
$$

we see that the Jack polynomials correspond to the case $a=-\varrho, b=\varrho+2 / \alpha$ and $c=1 / \alpha+1 / 2$.

From Erdélyi et al. (1981), we know that a solution of (8) which is regular at $w=0$ is given by

$$
f(w)=\sum_{n=0}^{\infty} \frac{(a)_{n}(b)_{n}}{(c)_{n} n !} w^{n}={ }_{2} F_{1}(a, b ; c ; w),
$$


where ${ }_{2} F_{1}(a, b ; c ; w)$ is the classical hypergeometric function, which in what follows will be denoted as $F(a, b ; c ; w)$.

Thus a solution of (7) is

$$
f(z)=F\left(-\varrho, \varrho+\frac{2}{\alpha} ; \frac{1}{\alpha}+\frac{1}{2} ; \frac{1-z}{2}\right) .
$$

Let us refine the above solution by applying properties of the hypergeometric functions. From Erdélyi et al. (1981, Section 2.11, p. 111, equation (2)), we see that

so

$$
F\left(2 d, 2 e ; d+e+\frac{1}{2} ; t\right)=F\left(d, e ; d+e+\frac{1}{2} ; 4 t(1-t)\right),
$$

(9) $f(z)=F\left(-\varrho, \varrho+\frac{2}{\alpha} ; \frac{1}{\alpha}+\frac{1}{2} ; \frac{1-z}{2}\right)=F\left(-\frac{\varrho}{2}, \frac{\varrho}{2}+\frac{1}{\alpha} ; \frac{1}{\alpha}+\frac{1}{2} ; 1-z^{2}\right)$.

By Erdélyi et al. (1981, Section 2.10, p. 108, equation (1)),

$$
\begin{aligned}
F(a, b ; c ; t)= & A_{1} F(a, b ; a+b-c+1 ; 1-t) \\
& +A_{2}(1-t)^{c-a-b} F(c-a, c-b ; c-a-b+1 ; 1-t),
\end{aligned}
$$

where

$$
A_{1}=\frac{\Gamma(c) \Gamma(c-a-b)}{\Gamma(c-a) \Gamma(c-b)} \quad \text { and } \quad A_{2}=\frac{\Gamma(c) \Gamma(a+b-c)}{\Gamma(a) \Gamma(b)} .
$$

Thus (9) can be written as

$$
\begin{aligned}
F\left(-\frac{\varrho}{2}, \frac{\varrho}{2}+\frac{1}{\alpha} ; \frac{1}{\alpha}+\frac{1}{2} ; 1-z^{2}\right)= & A_{1} F\left(-\frac{\varrho}{2}, \frac{\varrho}{2}+\frac{1}{\alpha} ; \frac{1}{2} ; z^{2}\right) \\
& +A_{2} z F\left(\frac{1}{\alpha}+\frac{1+\varrho}{2}, \frac{1}{2}-\frac{\varrho}{2} ; \frac{3}{2} ; z^{2}\right),
\end{aligned}
$$

where

$$
A_{1}=\frac{\Gamma\left(\frac{1}{\alpha}+\frac{1}{2}\right) \Gamma\left(\frac{1}{2}\right)}{\Gamma\left(\frac{1}{\alpha}+\frac{1+\varrho}{2}\right) \Gamma\left(\frac{1-\varrho}{2}\right)} \quad \text { and } \quad A_{2}=\frac{\Gamma\left(\frac{1}{\alpha}+\frac{1}{2}\right) \Gamma\left(-\frac{1}{2}\right)}{\Gamma\left(-\frac{\varrho}{2}\right) \Gamma\left(\frac{\varrho}{2}+\frac{1}{\alpha}\right)} .
$$

Then the Jack polynomials of second order are given by

$$
\begin{aligned}
& \text { (10) } \frac{C_{\left(k_{1}, k_{2}\right)}^{(\alpha)}(Y)}{C_{\left(k_{1}, k_{2}\right)}^{(\alpha)}\left(I_{2}\right)} \\
& =\frac{\left(y_{1} y_{2}\right)^{\left(k_{1}+k_{2}\right) / 2} \Gamma\left(\frac{1}{\alpha}+\frac{1}{2}\right) \Gamma\left(\frac{1}{2}\right)}{\Gamma\left(\frac{1}{\alpha}+\frac{1+\varrho}{2}\right) \Gamma\left(\frac{1-\varrho}{2}\right)} F\left(-\frac{\varrho}{2}, \frac{\varrho}{2}+\frac{1}{\alpha} ; \frac{1}{2} ; \frac{\left(y_{1}+y_{2}\right)^{2}}{4 y_{1} y_{2}}\right) \\
& +\frac{\left(y_{1} y_{2}\right)^{\left(k_{1}+k_{2}-1\right) / 2}}{2\left(y_{1}+y_{2}\right)^{-1}} \frac{\Gamma\left(\frac{1}{\alpha}+\frac{1}{2}\right) \Gamma\left(-\frac{1}{2}\right)}{\Gamma\left(-\frac{\varrho}{2}\right) \Gamma\left(\frac{\varrho}{2}+\frac{1}{\alpha}\right)} F\left(\frac{1}{\alpha}+\frac{1+\varrho}{2}, \frac{1}{2}-\frac{\varrho}{2} ; \frac{3}{2} ; \frac{\left(y_{1}+y_{2}\right)^{2}}{4 y_{1} y_{2}}\right) .
\end{aligned}
$$


Since $\varrho$ is a nonnegative integer, (10) can be simplified by considering the hypergeometric functions with $\varrho$ being either even or odd. To distinguish the case under consideration, odd or even, we will mark $A_{1}$ and $A_{2}$ with the upper indices $o$ or $e$. Observing that

- $\Gamma(1 / 2+z) \Gamma(1 / 2-z)=\pi \sec (\pi z)$,

- $\Gamma(z) \Gamma(-z)=-\pi z^{-1} \csc (\pi z)$,

- $\Gamma(z+n)=z(z+1)(z+2) \cdots(z+n-1) \Gamma(z)$,

the following results are obtained:

Even case. If $\varrho=k_{1}-k_{2}=2 n, n=0,1,2, \ldots$, then

$$
A_{1}^{e}=\frac{(-1)^{n} \prod_{i=0}^{n-1}(1+2 i)}{\prod_{i=0}^{n-1}(1+2(1 / \alpha+i))} \quad \text { and } \quad A_{2}^{e}=0 .
$$

Odd case. If $\varrho=k_{1}-k_{2}=2 n+1, n=0,1,2, \ldots$, then

$$
A_{1}^{o}=0 \quad \text { and } \quad A_{2}^{o}=(2 n+1) A_{1}^{e} .
$$

Three particular cases are of interest in the literature: the quaternionic case $(\alpha=1 / 2)$, the complex zonal polynomials $(\alpha=1)$ and the real zonal polynomials $(\alpha=2)$. These results are summarised in the following table:

\begin{tabular}{ccccccc}
\hline$\alpha$ & $\varrho$ & $a$ & $b$ & $c$ & $A_{1}$ & $A_{2}$ \\
\hline \multirow{2}{*}{$\frac{1}{2}$} & even & $-n$ & $n+2$ & $\frac{1}{2}$ & $\frac{(-1)^{n} 3}{(2 n+1)(2 n+3)}$ & 0 \\
& odd & $n+3$ & $-n$ & $\frac{3}{2}$ & 0 & $\frac{(-1)^{n} 3}{(2 n+3)}$ \\
\hline & even & $-n$ & $n+1$ & $\frac{1}{2}$ & $\frac{(-1)^{n}}{(2 n+1)}$ & 0 \\
1 & & & & $\frac{3}{2}$ & 0 & $(-1)^{n}$ \\
\hline & odd & $n+2$ & $-n$ & $\frac{1}{2}$ & $\frac{(-1)^{n}(2 n) !}{2^{2 n}(n !)^{2}}$ & 0 \\
2 & even & $-n$ & $n+1 / 2$ & $\frac{1}{2}$ & 0 & $\frac{(-1)^{n}(2 n+1) !}{2^{2 n}(n !)^{2}}$ \\
\hline
\end{tabular}

Finally, since $F(a, b ; c ; z)=F(b, a ; c ; z)$, the above formula for the real zonal polynomials corresponds to that derived by James (1968).

Acknowledgments. The authors wish to express their gratitude to the editor and the referees for their helpful comments and suggestions. This research work was partially supported by CONACYT-México, research grant no. 45974-F. 


\section{References}

J. A. Díaz-García and F. J. Caro-Lopera (2006), An alternative approach for deriving the Laplace-Beltrami operator for the zonal polynomials of positive semidefinite and definite matrix argument, Far East J. Math. Sci. 22, 273-290.

J. A. Díaz-García and F. J. Caro-Lopera (2007), Derivation of the Laplace-Beltrami operator for the zonal polynomials of positive definite Hermitian matrix argument, Appl. Math. Sci. 1, 191-200.

I. Dumitriu, A. Edelman and G. Shuman (2005), MOPSJ: Multivariate orthogonal polynomials (symbolically), Mathworld, http://mathworld.wolfram.com/.

A. Erdélyi et al. (1981), Higher Transcendental Functions 1, Robert E. Krieger, Malabar.

I. P. Goulden and D. M. Jackson (1996), Connection coefficients, matchings, maps and combinatorial conjectures for Jack symmetric functions, Trans. Amer. Math. Soc. 348, 873-892.

K. I. Gross and D. St. P. Richards (1987), Special functions of matrix argument. I: Algebraic induction, zonal polynomials and hypergeometric functions, ibid. 301, 781-811.

A. T. James (1964), Distributions of matrix variate and latent roots derived from normal samples, Ann. Math. Statist. 35, 475-501.

A. T. James (1968), Calculation of zonal polynomial coefficients by use of the LaplaceBeltrami operator, ibid. 39, 1711-1718.

C. G. Khatri (1970), On the moments of traces of two matrices in three situations for complex multivariate normal populations, Sankhyā A 32, 65-80.

P. Koev (2004), http://math.mit.edu/ plamen.

P. Koev and A. Edelman (2006), The efficient evaluation of the hypergeometric function of matrix argument, Math. Comp. 75, 833-846.

R. J. Muirhead (1982), Aspects of Multivariate Statistical Theory, Wiley, New York.

P. Sawyer (1997), Spherical functions on symmetric cones, Trans. Amer. Math. Soc. 349, 3569-3584.

Francisco J. Caro-Lopera

Department of Basic Mathematics

Centro de Investigación en Matemáticas

Callejón de Jalisco s/n

Mineral de Valenciana

36240 Guanajuato, Guanajuato, México

E-mail: fjcaro@cimat.mx

Graciela González-Farías

Department of Statistics

Centro de Investigación en Matemáticas

Callejón de Jalisco s/n

Mineral de Valenciana

36240 Guanajuato, Guanajuato, México

E-mail: farias@cimat.mx
José A. Díaz-García

Department of Statistics and Computation Universidad Autónoma Agraria Antonio Narro 25315 Buenavista Saltillo, Coahuila, México E-mail: jadiaz@uaaan.mx

Received on 1.3.2006;

revised version on 22.2.2007 
